\title{
A Preface on Aims
}

This book is a unified collection of essays in the rhetoric of the sciences and technologies discussing a multitude of ways of framing and modeling. The book is organized around a set of themes embedded in a listed set of quotes in the appendix that encapsulates my thoughts on simplifying complexity and the consequent oversimplifications of the simplifications made of the apparent complexity.

These essays center on three basic concerns about my own analytic and interpretive methods that I recently developed in reflecting on the complexity that I found in my own experiences in using different frames of knowledge that I have encountered in my overall long life experiences and my sustained inquiries into the methods that I once used 1) in the teaching of high school sciences, 2) in my teaching of rhetoric in undergraduate communications studies course work, and that 3) I used in a variety discipline areas in the teaching of philosophy. These three methods are based on three narratives that illustrate for me my present way of looking at the way we communicate with each other from a perspective that I have adopted, which was named and modeled by a good friend Preston Covey, and labeled by him as 'the wells of ignorance'.

The narratives of these experiences illustrate three propositions about scientific, humanistic, and rhetorical communication:

- The interpretation of what we think, say, and do is a matter of context and background knowledge. (Dan Sperber)

- We negotiate with others what we think is knowledge by making appeals and responses to each other by noting and reminding what we think we know. (Ludwig Wittengenstein)

- We use conceptual dyads and triads rhetorically to interpret as best we can clearly and simply what we think we know and understand. (George E. Yoos)

Coming to terms with ignorance, I suggest, is the key to understanding what we think of as knowledge. It is gained through the processes of falsification of each other's beliefs. We gain understanding through our oral interpretation of visual script and frames and models that are purported to be representations of knowledge claims. The aim of the book is that through the use of visual frames and models and through lists of contrasting and correlative distinctions it can be shown how we can create new language and new forms of graphic representations.

We use these frames and models as prosthetic, mnemonic, and attention controlling devices to produce simpler perspectives on the complications that we find in trying to understand different forms of complexity. We use these graphic forms of language to overcome our mnemonic and structuring limitations in organizing what we need to do to escape our existential ignorance (our wells of ignorance). 
The book as a whole aims to display some of the means that we have of creating visual language and graphic modes that we need to simplify the complexity that we find in the different sciences, both social and physical, that we find evolving at various times and in different places and in different cultures. The book continues my attempt to explain what I have argued about before about the value of rhetoric and communications in trying to make sense about the puzzles that others find in talking about what is real and about what are the true reality based structures to be found in our world.

My personal view of life is that it is about empowerment, the elimination of ignorance, and about the joy and beauty that we find existing in the different forms of life. Thus the sciences, both social and natural, are ultimately about conceptions of values and power (Flyvbjerg, 2001) that are directing and shaping our social practices and shaping our personal forms of expertise that we use to enhance the qualities of our lives practically.

I am attempting to illustrate the creative aspects of modeling and to show how the uses of models are best interpreted and understood by looking at the sciences and technologies as plural, not as systematically explainable or derivable from any unified theoretical representations such as the many proposed Theories of Everything (TOES) try to do. Such devising of unified theoretical representations to my mind is a favorite metaphysical pursuit in our modern age especially to be found among theoretical physicists and astronomers.

Specifically, the book attempts to do this by surveying the different models that have evolved out of the innate biological development of the frames of grammar, logic, and the modes of orientation, and especially through the evolution of biological and socially constructed systems of numerosity and measurement that evolved and developed in the rigors of science in the different formal languages used in the various sciences and technologies.

Essentially, the book is an exercise in genealogy much in the spirit of Nietzsche and Foucault. It is about the archeology or the anthropology of knowledge based upon the study of error that historically has progressively been eclipsed and has been eliminated rationally by discovering the numerous falsehoods to be found in our cultural mythical origins. There has been a dramatic increase in what now is thought to be available as public knowledge. The book attempts to show how human action is centered in practice (praxis). It is centered in the human artifice of the social constructions that we have developed as the rules of thumb that human beings use to solve their practical problems of living.

The book is about how the plurality of the sciences and technologies that develop around the prosthetics of printed language and visual models. These help us create new models and new modes of language to help us understand and to solve our problems. In doing so the models and frames that we create become rhetorical tools in scientific and technological communication. Our understanding of some of these rhetorical tools whereby we simplify complexity is key to our clarification of 
X - A Preface on Aims

the communication that is used 1) in the languages of the humanities, 2) in the new language of the sciences, and 3) in the languages of new and evolving growing forms of expertise.

George E. Yoos

Clear Lake, Minnesota, USA

May 2012 